\title{
Staphylococcus lugdunensis: antimicrobial susceptibility and optimal treatment options
}

\author{
Lana Taha $^{1} \cdot$ Marc Stegger $^{2} \cdot$ Bo Söderquist ${ }^{1,3}$ \\ Received: 20 March 2019 / Accepted: 25 April 2019 /Published online: 29 May 2019 \\ (C) The Author(s) 2019
}

\begin{abstract}
Staphylococcus lugdunensis is a coagulase-negative staphylococcus (CoNS) with unusual pathogenicity resembling that of S. aureus. Unlike other CoNS, S. lugdunensis remains susceptible to most antibiotics. The resistance to penicillin varies widely (range, 15-87\% worldwide), whereas methicillin resistance is still rare. We aimed to evaluate treatment options for infections caused by $S$. lugdunensis and more specifically to investigate whether penicillin G could be a better treatment choice than oxacillin. Susceptibility testing was performed using the disc diffusion method for penicillin G, cefoxitin, trimethoprim/sulfamethoxazole, erythromycin, clindamycin, gentamicin, norfloxacin, fusidic acid, rifampicin, and fosfomycin. Isolates susceptible to penicillin $\mathrm{G}$ were further tested with a gradient test for penicillin $\mathrm{G}$ and oxacillin. Of the 540 clinical isolates tested, $74.6 \%$ were susceptible to penicillin G. Among these penicillin-susceptible isolates, the $\mathrm{MIC}_{50}$ and $\mathrm{MIC}_{90}$ values for penicillin $\mathrm{G}$ were threefold lower than that for oxacillin. A majority of the isolates were susceptible to all other antibiotics tested. Breakpoints for fosfomycin have not yet been defined, and so no conclusions could be drawn. Two isolates were resistant to cefoxitin and carried the mecA gene; whole-genome sequencing revealed that both harbored the SCCmec element type IVa(2B). S. lugdunensis isolated in Sweden were susceptible to most tested antibiotics. Penicillin G may be a more optimal treatment choice than oxacillin. Although carriage of the mecA gene is rare among $S$. lugdunensis, it does occur.
\end{abstract}

Keywords Staphylococcus lugdunensis $\cdot$ Antibiotic susceptibility testing $\cdot$ Penicillin $G \cdot$ Oxacillin

\section{Introduction}

Staphylococcus lugdunensis is a coagulase-negative staphylococcus (CoNS) with a significant pathogenic potential compared with other CoNS. First described in 1988 [1], it is part of the normal skin flora and is most commonly found in the groin area, lower extremities, and axilla [2,3], that is, in areas

Electronic supplementary material The online version of this article (https://doi.org/10.1007/s10096-019-03571-6) contains supplementary material, which is available to authorized users.

Bo Söderquist

bo.soderquist@oru.se

1 School of Medical Sciences, Faculty of Medicine and Health, Örebro University, Örebro, Sweden

2 Department of Bacteria, Parasites and Fungi, Statens Serum Institut, Copenhagen, Denmark

3 Department of Laboratory Medicine, Clinical Microbiology, Örebro University Hospital, SE-701 85 Örebro, Sweden possessing excess apocrine sweat glands [4]. However, it can also be frequently found in the nasal cavity [5]. Although a commensal, S. lugdunensis has the ability to cause aggressive infections, resembling those of $S$. aureus rather than of other CoNS. S. lugdunensis is a common cause of skin and soft tissue infections $[4,6,7]$, but can also cause bone and joint infections [8], native and prosthetic valve endocarditis $[9,10]$, and bacteremia $[11,12]$.

S. lugdunensis remains remarkably susceptible to most antibiotics, unlike many other CoNS such as S. epidermidis. Resistance to penicillin has been reported to be as low as $15.5 \%$ and $20 \%$ in Sweden and Denmark, respectively [4, 13], but considerably higher in the USA (45\%) [14] and Taiwan (87\%) [15]. Resistance to erythromycin and clindamycin is overall very low [4, 14]; Taiwan is the exception, with reported resistance levels of $17-25 \%[15,16]$. According to a review by Argemi et al. [17], susceptibility to fosfomycin is greatly variable, reporting resistance in $>$ $50 \%$ of isolates. However, breakpoints for fosfomycin have not yet been defined. The prevalence of methicillin resistance and presence of the $m e c A$ gene ranges from 0 to $8.3 \%$ [14, 
18-21], with the exception again being Taiwan, where the reported occurrence is $21 \%$ [16]. For detection of $m e c A$, cefoxitin disc breakpoints are a better option than oxacillin disc breakpoints, which have lower sensitivity and specificity [22]. The breakpoints for cefoxitin and oxacillin are the same as for $S$. aureus (www.eucast.org).

Until recently, the methods used to distinguish different CoNS species from S. aureus were based on phenotypical properties, such as the DNase and coagulase tests [9]. Moreover, the differentiation between $S$. lugdunensis and other staphylococci has been based on the presence of ornithine decarboxylase activity (ODC) and a pyrrolidonyl arylamidase test $[14,22]$ that shows positivity in more than $90 \%$ of $S$. lugdunensis isolates [23]. However, although $S$. lugdunensis does not produce secreted coagulase, it can sometimes be mistaken for $S$. aureus at clinical microbiology laboratories. This is explained by the fact that up to $65 \%$ [24] of S. lugdunensis isolates possess a membrane-bound form of coagulase (clumping factor) that shows a positive result in the latex agglutination test and slide coagulase test $[1,9,23]$. Since the implementation of MALDITOF MS, the identification of S. lugdunensis has become much easier, more accurate, and more cost effective for routine laboratories [25].

The first line of antimicrobial treatment of staphylococcal skin and soft tissue infections is isoxazolyl penicillin [26, 27], especially in areas with a low prevalence of MRSA. In Sweden, the prevalence of MRSA in blood cultures displaying positivity for S. aureus is still approximately $1 \%$. In addition, if a majority of $S$. lugdunensis also displays full susceptibility to penicillin, it would be of interest to evaluate whether penicillin could be a better treatment option than oxacillin for those penicillin-susceptible isolates, in order to optimize the treatment for infections caused by $S$. lugdunensis and thereby reduce selection of isoxazolyl penicillin-resistant staphylococci. We therefore aimed to compare the MIC values for penicillin and isoxazolyl penicillin in penicillin-susceptible isolates of S. lugdunensis and to determine the antibiotic susceptibility pattern of $S$. lugdunensis for multiple antimicrobial agents.

\section{Materials and methods}

\section{Bacterial isolates}

From January 2010 to October 2014 and from November 2017 to January 2018, all isolates that were identified as S. lugdunensis from clinical samples at the Department of Laboratory Medicine, Clinical Microbiology, Örebro University Hospital, Sweden, were saved. MALDI-TOF MS (Microflex LT and Biotyper 3.1, Bruker Daltonik, Bremen, Germany) was implemented at this department in January 2014 , which made it possible to perform an accurate identification of $S$. lugdunensis; before this, staphylococcal species were identified by screening with DNase and coagulase tests. The presence of ODC was tested in order to distinguish $S$. lugdunensis from other CoNS, and since some isolates of $S$. epidermidis also show positivity for ODC, further testing was performed with trehalose-mannitol broth to distinguish S. lugdunensis from S. epidermidis. Final species determination was in some cases performed by using API ID32 Staph (bioMérieux, Marcy l'Etoile, France).

All isolates were stored at $-80^{\circ} \mathrm{C}$ in preservation medium (Trypticase Soy Broth, BD Diagnostic Systems, Sparks, MD, USA, supplemented with $0.3 \%$ yeast extract, BD Diagnostic Systems, and 29\% horse serum, SVA, Uppsala, Sweden) and subcultured overnight at $36^{\circ} \mathrm{C}$ on Mueller-Hinton agar (BD Diagnostic Systems). Isolates that did not have the phenotypical appearance, color, or typical smell of S. lugdunensis were retested with MALDI-TOF MS.

\section{Susceptibility testing}

Susceptibility testing for ten antibiotics was performed with the disc diffusion method according to the 2017 EUCAST guidelines [28]. The cultures were suspended in sterile saline to $0.5 \mathrm{McF}$ arland and inoculated on Mueller-Hinton agar plates (BD Diagnostic Systems). A maximum of three antibiotic discs per agar plate were put in place and inoculated for $18 \mathrm{~h} \pm 2 \mathrm{~h}$ at $35^{\circ} \mathrm{C}$. Ten antibiotics were tested (all discs from Oxoid, Basingstoke, UK): penicillin G (1 IU), cefoxitin $(30 \mu \mathrm{g})$, clindamycin $(2 \mu \mathrm{g})$, norfloxacin $(10 \mu \mathrm{g})$, trimethoprim-sulfamethoxazole $(25 \mu \mathrm{g})$, erythromycin $(15 \mu \mathrm{g})$, gentamicin $(10 \mu \mathrm{g})$, fusidic acid $(10 \mu \mathrm{g})$, rifampicin $(5 \mu \mathrm{g})$, and fosfomycin $(50 \mu \mathrm{g})$. Cefoxitin $(30 \mu \mathrm{g})$ discs were used as a screening test for oxacillin resistance.

All isolates susceptible to penicillin $\mathrm{G}$ were further tested with a gradient test (Etest, bioMérieux) for penicillin $\mathrm{G}$ and oxacillin, also according to EUCAST guidelines. Identification of the mecA gene was performed on those isolates resistant to cefoxitin, using Genie II and eazyplex MRSAplus (Amplex Diagnostics GmbH, München, Germany).

\section{WGS and analysis of SCCmec}

Genomic DNA was extracted from the $m e c A$-positive isolates using the DNeasy Blood and Tissue Kit (Qiagen, Hilden, Germany), with subsequent library construction using the Nextera XT Kit (Illumina, Little Chesterford, UK) before whole-genome sequencing was performed using a 300-cycle kit on the NextSeq platform (Illumina) according to the manufacturer's instructions. Resistance genes were identified with ARIBA [29], using raw reads against the ResFinder database (https://cge.cbs.dtu.dk/services/ResFinder/). The raw reads were assembled using version 3.10 .1 of SPAdes [30] and 
used for MLST typing with the MLST command-line tool (https://github.com/tseemann/mlst). SCCmec types were identified using version 1.2 of SCCmecFinder [31].

\section{Results}

The samples included in the present study were mainly from primary and secondary infections of skin and soft tissue structures and consisted of 569 isolates. Of these, 29 isolates were either non-viable or had been misidentified as $S$. lugdunensis. The site of sampling, etiology of the misidentified isolates, and number of isolates included from each year are given in Supplementary files (Tables S1A-S3A). Antibiotic susceptibility testing on the remaining 540 isolates revealed that $25.4 \%$ (137/ 540) were resistant to penicillin G (Table 1). Furthermore, there was also a trend towards decreasing resistance during the time period (chi-squared test for trend; $p<0.001$; Table S3A). Two isolates that were resistant to cefoxitin (oxacillin MIC $>256 \mathrm{mg}$ ) $\mathrm{L}$ and $32 \mathrm{mg} / \mathrm{L}$ respectively) and carried the mecA gene were whole-genome sequenced in order to characterize the SCCmec (see below). One isolate was resistant to norfloxacin but remained susceptible to all other antibiotics. Of the two isolates that were resistant to rifampicin, one was also resistant to trimethoprim-sulfamethoxazole; and of the two isolates resistant to gentamicin, one was also resistant to fusidic acid. A total of 34 isolates were resistant to erythromycin, and all but one also showed resistance to clindamycin. Zone diameters for all antibiotics tested by the disc diffusion method are presented in Fig. 1. Although as previously mentioned there are as yet no defined breakpoints for fosfomycin, the median of the zone diameter was $28 \mathrm{~mm}$ and $85.7 \%$ of the isolates had a zone diameter larger than $20 \mathrm{~mm}$ (Fig. 1).

The MIC values determined by a gradient test for penicillin $\mathrm{G}$ were all lower than (or the same as) those for oxacillin (Fig. 2). $\mathrm{MIC}_{50}$ was $0.064 \mathrm{mg} / \mathrm{L}$ for penicillin $\mathrm{G}$ and

Table 1 Antibiotic susceptibility pattern of 540 Staphylococcus lugdunensis isolates tested with the disc diffusion method

\begin{tabular}{ll}
\hline Antibiotic & No. (\%) susceptible \\
\hline Penicillin G & $403(74.6)$ \\
Gentamicin & $538(99.6)$ \\
Rifampicin & $538(99.6)$ \\
Cefoxitin & $538(99.6)$ \\
Fusidic acid & $528(97.8)$ \\
Trimethoprim/sulfamethoxazole & $539(99.8)$ \\
Norfloxacin & $539(99.8)$ \\
Clindamycin & $494(91.5)$ \\
Erythromycin & $506(93.7)$ \\
\hline
\end{tabular}

$0.5 \mathrm{mg} / \mathrm{L}$ for oxacillin, whereas $\mathrm{MIC}_{90}$ was $0.094 \mathrm{mg} / \mathrm{L}$ for penicillin $\mathrm{G}$ and $0.75 \mathrm{mg} / \mathrm{L}$ for oxacillin. In general, the MIC for penicillin $\mathrm{G}$ was three dilution steps lower than that for oxacillin ( $p<0.0001$ paired $t$ test). Whole-genome sequencing of the two mecA-positive $S$. lugdunensis to an average depth of 100 or above revealed that they were ST38 and ST44 according to the MLST typing scheme. Both isolates harbored the SCCmec type IVa(2B), but with variations within the $\mathrm{J} 3$ region. Further analysis of the resistome identified $\operatorname{erm}(\mathrm{C})$, tet $(\mathrm{K})$, and blaZ in the ST38 isolate, whereas the ST44 only carried blaZ in addition to mecA. These findings were in concordance with the phenotypic results.

\section{Discussion}

As a coagulase-negative staphylococcus, $S$. lugdunensis remains remarkably susceptible to various antimicrobial agents. In the present study, conducted in Örebro, Sweden, the clinical S. lugdunensis isolates were almost fully susceptible to all tested antibiotics. However, resistance levels reported from other parts of the world indicate the emergence of resistance. A Taiwanese study reported resistances to penicillin and oxacillin of $87 \%$ and $20 \%$, respectively [15], while another study from the USA found that $45 \%$ of S. lugdunensis isolates were resistant to penicillin [14]. By comparison, our results showed a resistance level of $25.4 \%$ regarding penicillin $\mathrm{G}$, indicating that the isolates in our study remain mainly naïve or of wild type. Furthermore, there was rather a trend towards decreasing resistance levels throughout the sample period. However, this species has the potential to become increasingly resistant, predominantly by acquiring the blaZ gene encoding penicillinase production, but other similar resistance mechanisms could be acquired. In addition, a case report by Kragsbjerg et al. [32] described the development of resistance to rifampicin and ciprofloxacin during treatment of a persistent $S$. lugdunensis infection in a patient with septic arthritis, infective endocarditis, and vertebral osteomyelitis.

Despite the fact that $S$. lugdunensis is susceptible to oxacillin in vitro, treatment failure is not uncommon [33], indicating that oxacillin may be suboptimal in some cases. As previous studies have shown, the prevalence of mecA, and thereby methicillin resistance, is not yet extensive among S. lugdunensis [14, 17-21]. The identification of SCCmec in two highly distant ST types provides evidence of multiple acquisitions, and whereas the whole-genome sequencing indicated overall the same type of SCC element (type IVa(2B)), they were of different origin based on their $\mathrm{J} 3$ differences. Regarding the other resistance genes in the two multidrugand methicillin-resistant $S$. lugdunensis isolates, analyses revealed a chromosomal integration of blaZ based on coverage, whereas the $\operatorname{tet}(\mathrm{K})$ and $\operatorname{erm}(\mathrm{C})$ were both carried on a small $4.5 \mathrm{~kb}$ plasmid previously found in both Staphylococcus 
Fig. 1 Zone diameters in millimeters (x-axis) for all antimicrobial agents tested against 540 Staphylococcus lugdunensis isolates using the disc diffusion method
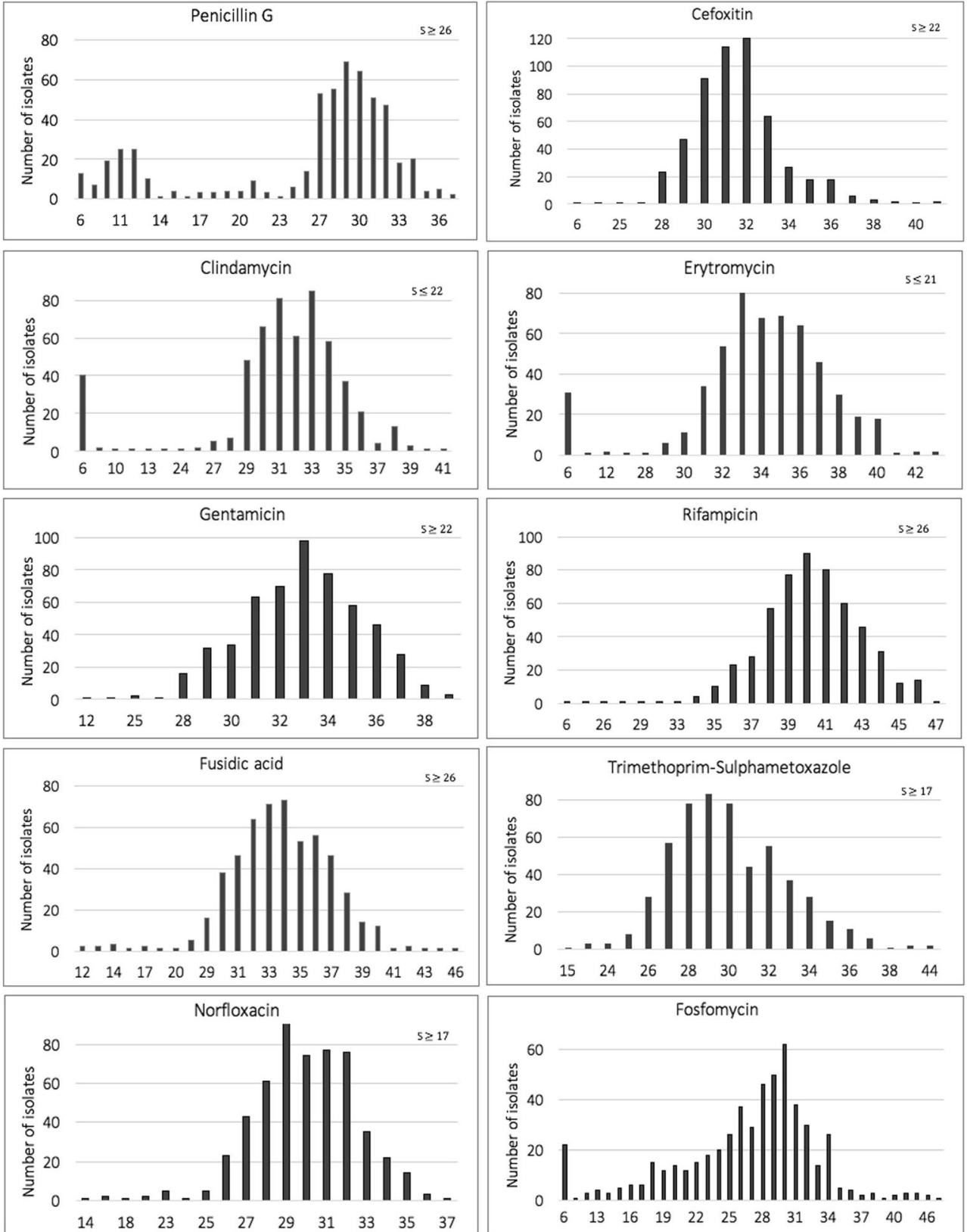

Fig. 2 Minimum inhibitory concentration $(\mathrm{mg} / \mathrm{L})$ values for penicillin $\mathrm{G}$ and oxacillin in the 403 penicillin G-susceptible isolates among all 540 Staphylococcus lugdunensis isolates tested with the disc diffusion method. Etest was used for gradient testing.

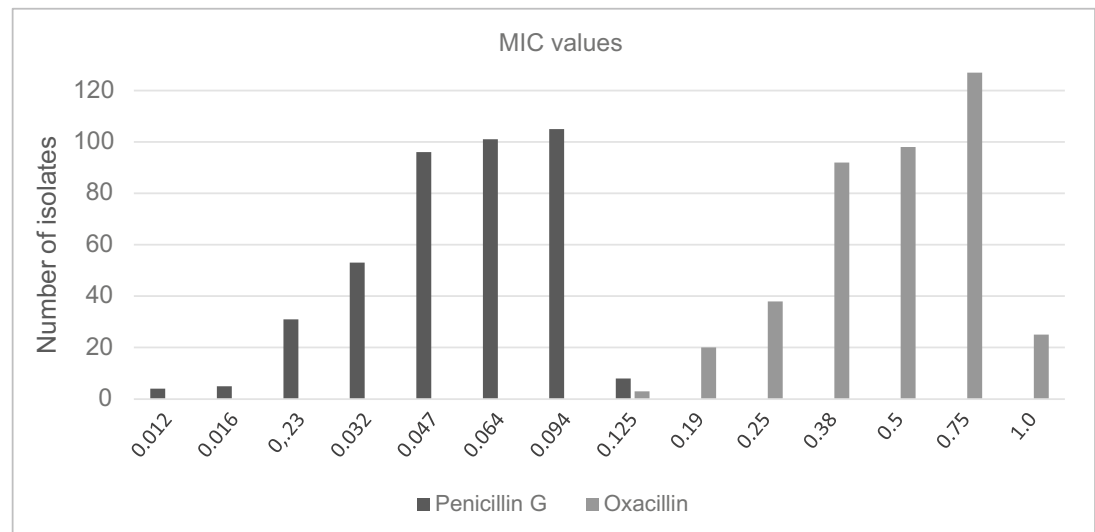


aureus strain USA300 FPR3757 [34] and Staphylococcus epidermidis strain ATTC 12228 [35].

As noted in the present study, penicillin-susceptible S. lugdunensis displayed lower MIC values for penicillin G than for oxacillin, with a difference of approximately three dilution steps between their $\mathrm{MIC}_{50}$ as well as the highest MIC values. From a clinical point of view, this implies that a lower concentration (i.e., lower dosage) of penicillin $\mathrm{G}$ is needed for a therapeutic effect in comparison with oxacillin. Unnecessarily high doses of antibiotics may not only increase the risk of sideeffects in individual patients but could also have adverse effects on the macro- and microenvironment such as the skin and the gut microbiome. Differences in pharmacokinetics offer another reason why penicillin $\mathrm{G}$ could be a better choice of treatment. Isoxazolyl penicillin has a higher serum protein binding capacity (94-98\%) than penicillin G (65\%) [36], which means that higher doses of the drug are needed to reach therapeutic levels in order to achieve optimal concentrations of a free fraction of the antimicrobial agent at the infection site since antibacterial efficacy is correlated to $\mathrm{T}>\mathrm{MIC}$.

Regarding fosfomycin, resistance among S. lugdunensis has only been described in a single paper [17]. Furthermore, there are no current international or national breakpoints for fosfomycin. Our results did not show a distinct difference between a supposed wild population and isolates displaying decreased susceptibility or resistance. When tested for fosfomycin, the two sequenced isolates displayed zone diameters of $20 \mathrm{~mm}$ and $26 \mathrm{~mm}$ respectively, and neither of them harbored $f \circ s A / f o s B$ genes.

One limitation of the present study is that no data were available regarding the clinical course of disease or the severity of the infections. This limitation could be addressed in a prospective randomized trial comparing the efficacy of penicillin G/V versus cloxacillin/flucloxacillin.

The method used in this study is based on a standardized protocol and was performed according to the EUCAST guidelines. All laboratory work and the determinations were performed by one individual, eliminating the potential bias of performing and interpreting the susceptibility tests and gradient tests differently, and thus reducing inter-person variability. The major strength of this study lies in the number of isolates investigated, predominantly from skin and soft tissue infections, and the inclusion of present-day isolates.

Although knowledge has increased dramatically in the past decade, investigations into the diversity, virulence, and population structure of $S$. lugdunensis are still in their infancy. Like other CoNS, S. lugdunensis has the ability to produce biofilm in various degrees, and despite being highly susceptibility to most antimicrobial agents, once biofilm is produced an infection becomes significantly more difficult to treat [18]. However, despite the presence of the icaADBC locus, which encodes polysaccharides central in biofilm production in most staphylococcal species, it is not used in the formation of biofilm by $S$. lugdunensis.
Instead, the biofilm displayed by this species is more proteinaceous [37]. The autolysin/adhesion AtlL plays a role in biofilm formation of $S$. lugdunensis but also acts as a virulence factor by binding to extracellular matrix proteins and by promoting internalization [38]. In addition, the surface protein sortase A seems to be important in the pathogenesis of infective endocarditis [39]. Another recent discovery by Zipperer et al. [40] is that some isolates of $S$. lugdunensis have the ability to produce lugdunin, a novel antibiotic compound that inhibits the growth of $S$. aureus, other Gram-positive bacteria, and even vancomycin-resistant enterococci. This finding could offer the possibility of development of new antimicrobial agents.

Our results indicate that penicillin is a better option than oxacillin for treating $S$. lugdunensis infections. A review of the literature reveals that a great majority of $S$. lugdunensis still remain susceptible to penicillin in the Western world. This is beneficial since penicillin $G$ is a narrow spectrum antibiotic which has advantageous pharmacokinetics as well as MIC values that are three-fold lower than oxacillin, as shown in the present study. This finding should advocate the inclusion of penicillin $\mathrm{G}$ as a routine for antibiotic susceptibility testing of suspected CoNS or verified $S$. lugdunensis by clinical microbiological laboratories.

\section{Conclusion}

In the present study, performed in Sweden, the majority of S. lugdunensis isolates were susceptible to all tested antibiotics. Almost $75 \%$ of the isolates were susceptible to penicillin G. Among these, the MIC values for penicillin G were threefold lower than that for oxacillin, indicating that penicillin $\mathrm{G}$ may be a better treatment choice; a conclusion which may also be supported by pharmacokinetic data. Carriage of the mecA gene among $S$. lugdunensis was rare.

Acknowledgements We are sincerely obliged to Cecilia Bergh for statistical advice.

\section{Compliance with ethical standards}

Conflict of interest The authors declare that they have no conflict of interest.

Ethical considerations The laboratory ID was anonymized before handling. Information about the date of sampling and site of sampling was available. Only subcultured bacterial isolates were stored; no tissue material or other biological material was kept. There was no personal data available, and so no possibility of tracking patients or connecting them to the samples.

Open Access This article is distributed under the terms of the Creative Commons Attribution 4.0 International License (http:// creativecommons.org/licenses/by/4.0/), which permits unrestricted use, distribution, and reproduction in any medium, provided you give appropriate credit to the original author(s) and the source, provide a link to the Creative Commons license, and indicate if changes were made. 


\section{References}

1. Freney J, Brun Y, Bes M et al (1988) Staphylococcus lugdunensis sp. nov. and Staphylococcus schleiferi sp. nov., two species from human clinical specimens. Int J Syst Bacteriol 38:168-172

2. Bieber L, Kahlmeter G (2010) Staphylococcus lugdunensis in several niches of the normal skin flora. Clin Microbiol Infect 16:385388

3. van der Mee-Marquet N, Achard A, Mereghetti L et al (2003) Staphylococcus lugdunensis infections: high frequency of inguinal area carriage. J Clin Microbiol 41:404-409

4. Böcher S, Tønning B, Skov RL et al (2009) Staphylococcus lugdunensis, a common cause of skin and soft tissue infections in the community. J Clin Microbiol 47:946-950

5. Kaspar U, Kriegeskorte A, Schubert T, Peters G, Rudack C, Pieper DH, Wos-Oxley M, Becker K (2016) The culturome of the human nose habitats reveals individual bacterial fingerprint patterns. Environ Microbiol 18:2130-2142

6. Arias M, Tena D, Apellániz M et al (2010) Skin and soft tissue infections caused by Staphylococcus lugdunensis: report of 20 cases. Scand J Infect Dis 42:879-884

7. Papapetropoulos N, Papapetropoulou M, Vantarakis A (2013) Abscesses and wound infections due to Staphylococcus lugdunensis: report of 16 cases. Infection 41:525-528

8. Douiri N, Hansmann Y, Lefebvre N et al (2016) Staphylococcus lugdunensis: a virulent pathogen causing bone and joint infections. Clin Microbiol Infect 22:747-748

9. Becker K, Heilmann C, Peters G (2014) Coagulase-negative staphylococci. Clin Microbiol Rev 27:870-926

10. Van Hoovels L, De Munter P, Colaert J et al (2005) Three cases of destructive native valve endocarditis caused by Staphylococcus lugdunensis. Eur J Clin Microbiol Infect Dis 24:149-152

11. Ebright JR, Penugonda N, Brown W (2004) Clinical experience with Staphylococcus lugdunensis bacteremia: a retrospective analysis. Diagn Microbiol Infect Dis 48:17-21

12. Choi S-H, Chung J-W, Lee EJ et al (2010) Incidence, characteristics, and outcomes of Staphylococcus lugdunensis bacteremia. J Clin Microbiol 48:3346-3349

13. Hellbacher C, Törnqvist E, Söderquist B (2006) Staphylococcus lugdunensis: clinical spectrum, antibiotic susceptibility, and phenotypic and genotypic patterns of 39 isolates. Clin Microbiol Infect 12:43-49

14. Kleiner E, Monk AB, Archer GL et al (2010) Clinical significance of Staphylococcus lugdunensis isolated from routine cultures. Clin Infect Dis 51:801-803

15. Yen T-Y, Sung Y-J, Lin H-C et al (2016) Emergence of oxacillinresistant Staphylococcus lugdunensis carrying staphylococcal cassette chromosome mec type V in central Taiwan. J Microbiol Immunol Infect 49:885-891

16. Lin J-F, Cheng C-W, Kuo A-J et al (2015) Clinical experience and microbiologic characteristics of invasive Staphylococcus lugdunensis infection in a tertiary center in northern Taiwan. J Microbiol Immunol Infect 48:406-412

17. Argemi X, Hansmann Y, Riegel P et al (2017) Is Staphylococcus lugdunensis significant in clinical samples? J Clin Microbiol 55: 3167-3174

18. Frank KL, Reichert EJ, Piper KE et al (2007) In vitro effects of antimicrobial agents on planktonic and biofilm forms of Staphylococcus lugdunensis clinical isolates. Antimicrob Agents Chemother 51:888-895

19. Ferreira RBR, Iorio NLP, Malvar KL et al (2003) Coagulasenegative staphylococci: comparison of phenotypic and genotypic oxacillin susceptibility tests and evaluation of the agar screening test by using different concentrations of oxacillin. J Clin Microbiol 41:3609-3614

20. Hussain Z, Stoakes L, Massey V et al (2000) Correlation of oxacillin MIC with mecA gene carriage in coagulase-negative staphylococci. J Clin Microbiol 38:752-754

21. Ho P-L, Leung SM-H, Chow K-H et al (2015) Carriage niches and molecular epidemiology of Staphylococcus lugdunensis and methicillin-resistant $S$. lugdunensis among patients undergoing long-term renal replacement therapy. Diagn Microbiol Infect Dis 81:141-144

22. Tan TY, Ng SY, He J (2008) Microbiological characteristics, presumptive identification, and antibiotic susceptibilities of Staphylococcus lugdunensis. J Clin Microbiol 46:2393-2395

23. Frank KL, Del Pozo JL, Patel R (2008) From clinical microbiology to infection pathogenesis: how daring to be different works for Staphylococcus lugdunensis. Clin Microbiol Rev 21:111-133

24. Argemi X, Prévost G, Riegel P et al (2017) VISLISI trial, a prospective clinical study allowing identification of a new metalloprotease and putative virulence factor from Staphylococcus lugdunensis. Clin Microbiol Infect 23:334.e1-334.e8

25. Patel R (2013) Matrix-assisted laser desorption ionization-time of flight mass spectrometry in clinical microbiology. Clin Infect Dis $57: 564-572$

26. Claesson B, Norman C (2009) Hud- och mjukdelsinfektioner i öppenvård - epidemiologi, bakteriologi och diagnostik [skin and soft tissue infections in ambulatory care - epidemiology, bacteriology and diagnosis]. Information från Läkemedelsverket 20:28-36. www.lakemedelsverket.se

27. Stevens DL, Bisno AL, Chambers HF et al (2014) Practice guidelines for the diagnosis and management of skin and soft tissue infections: 2014 update by the Infectious Diseases Society of America. Clin Infect Dis 59:e10-e52

28. European Committee on Antimicrobial Susceptibility Testing. EUCAST Disk Diffusion Method for Antimicrobial Susceptibility Testing - Version 6.0. http://www.eucast.org/fileadmin/src/media/ PDFs/EUCAST_files/Disk_test_documents/Version_5/Manual_v_ 6.0 EUCAST Disk Test final.pdf. Accessed 27 Jan 2018

29. Hunt M, Mather AE, Sánchez-Busó L et al (2017) ARIBA: rapid antimicrobial resistance genotyping directly from sequencing reads. Microb Genom 3:e000131

30. Bankevich A, Nurk S, Antipov D et al (2012) SPAdes: a new genome assembly algorithm and its applications to single-cell sequencing. J Comput Biol 19:455-477

31. Kaya H, Hasman H, Larsen J et al (2018) SCCmecFinder, a webbased tool for typing of staphylococcal cassette chromosome mec in Staphylococcus aureus using whole-genome sequence data. mSphere 3(1):e00612-e00617

32. Kragsbjerg P, Bomfim-Loogna J, Tornqvist E et al (2000) Development of antimicrobial resistance in Staphylococcus lugdunensis during treatment - report of a case of bacterial arthritis, vertebral osteomyelitis and infective endocarditis. Clin Microbiol Infect 6:496-499

33. (2009) Farmakologisk behandling av bakteriella hud- och mjukdelsinfektioner - ny rekommendation [Pharmacological treatment of bacterial skin and soft tissue infections - new recommendation]. Information från Läkemedelsverket 20:16-27. www. lakemedelsverket.se

34. Diep BA, Gill SR, Chang RF et al (2006) Complete genome sequence of USA300, an epidemic clone of community-acquired meticillin-resistant Staphylococcus aureus. Lancet 367:731-739

35. Zhang Y-Q, Ren S-X, Li H-L et al (2003) Genome-based analysis of virulence genes in a non-biofilm-forming Staphylococcus epidermidis strain (ATCC 12228). Mol Microbiol 49:1577-1593 
36. Referensgruppen för Antibiotikafrågor. Antibiotikakompendium [Antibiotics Compendium]. http://www.sls.se/globalassets/raf/ ovriga-dokument/antibiotikakompendium.pdf. Accessed 16 Feb 2018

37. Frank KL, Patel R (2007) Poly- $N$-acetylglucosamine is not a major component of the extracellular matrix in biofilms formed by icaADBC-positive Staphylococcus lugdunensis isolates. Infect Immun 75:4728-4742

38. Hussain M, Steinbacher T, Peters G, Heilmann C, Becker K (2015) The adhesive properties of the Staphylococcus lugdunensis multifunctional autolysin AtlL and its role in biofilm formation and internalization. Int J Med Microbiol 305:129-139
39. Heilbronner S, Hanses F, Monk IR, Speziale P, Foster TJ (2013) Sortase A promotes virulence in experimental Staphylococcus lugdunensis endocarditis. Microbiology 159:2141-2152

40. Zipperer A, Konnerth MC, Laux C et al (2016) Human commensals producing a novel antibiotic impair pathogen colonization. Nature 535:511-516

Publisher's note Springer Nature remains neutral with regard to jurisdictional claims in published maps and institutional affiliations. 